\title{
Online Mendelian Inheritance in Man
}

National Cancer Institute

\section{Source}

National Cancer Institute. Online Mendelian Inheritance in Man. NCI Thesaurus. Code C43519.

This database is a catalog of human genes and genetic disorders authored and edited by Dr. Victor A. McKusick and his colleagues at Johns Hopkins and elsewhere, and developed for the World Wide Web by NCBI, the National Center for Biotechnology Information. The database contains textual information and references. It also contains copious links to MEDLINE and sequence records in the Entrez system, and links to additional related resources at $\mathrm{NCBI}$ and elsewhere. 